\title{
Modeling of Particle Separation in Bends of Rectangular Cross-section
}

\author{
Bingtao Zhao \\ Department of Environmental Engineering, Donghua University \\ Shanghai 200051, People's Republic of China
}

\begin{abstract}
A new theoretical model for predicting particle separation efficiency in bends is developed based on the investigation of flow pattern, the critical particle size separation theory and the boundary layer separation theory. The radial particle concentration gradient, instead of the usually assumed uniform radial particle concentration within the bends, is considered in this mathematical model. The local particle concentration and the separation efficiency can be calculated on the base of a time-of-flight model in terms of the particle size distribution of the feed. The effects of curvature ratio and bend angle on the separation efficiency in bends are also evaluated by the model.
\end{abstract}

Key words: Bends, Separation Efficiency, Theoretical Model

\section{INTRODUCTION}

Bends are widely used in the field of air pollution control and gas-solid separation for aerosol sampling and industrial applications. In order to estimate the bends performance, the theories of particle separation are developed by many contributors using different methods with simplifying assumptions. Representative researches mainly include: Landahl and Harrmann [1] proposed a model for separation of aerosol particles in a curved pipe where the separation depended only on the Stokes number (Stk). Davis [2] used aerosol particles to visualize the secondary flow patterns in pipes with bend angle, ranging from $30^{\circ}$ to $180^{\circ}$, however, no data on the particles loss in the pipe were gathered. Cheng and Wang [3] developed a model for the impaction efficiency of aerosol particles in $90^{\circ}$ bends, this model was based on an analytical laminar flow solution and gave the separation efficiency as a function of the Stokes number and curvature ratio. Crane and Evans [4] performed numerical calculations to predict the behavior of aerosol particles in laminar flow in $90^{\circ}$ bends, their results showed reasonable agreement with those of Cheng and Wang [3]. Cheng and Wang [5] re-examined the separation of particles in the bends, they conclude that in the laminar flow regime, the particle separation was mainly a functions of Stokes number and flow Reynolds number $(\mathrm{Re})$ for curvature ratio between 4 to 20. Pui et al. [6] experimentally evaluated the separation of particle in $90^{\circ}$ bends, for turbulent flow, they suggested that the separation efficiency does not depend on either curvature ratio or Reynolds number but only Stokes number, and offered a correlation for evaluated the particle separation efficiency. Tsai and Pui [7] used a three-dimensional numerical procedure to examine the particle separation for laminar flow in a pipe with a $90^{\circ}$ bend, they found significant variation in the separation efficiency as a function of curvature ratio. Recently, McFarland et al. [8] presented a numerical simulation for aerosol particle separation in bends with turbulent flow, their results showed the curvature ratio and bend angle instead of Reynolds number sufficiently affects the particle separation.

In the present work, a new theoretical model for predicting particle separation efficiency in bends is developed based on the investigation of flow pattern, the critical particle size separation theory and the boundary layer separation theory, which considers a varied radial particle concentration gradient instead of a uniform radial concentration profile for uncollected particles within the bends. In addition, the effects of curvature ratio bend angle and flow rate on the separation efficiency are evaluated by the model.

Theory: The conventional bends geometry is shown in Fig. 1. To develop the new calculation model for determining the particle separation, the following assumptions are made: The particle is spherical in shape, the motion of a particle is not influenced by the presence of neighboring particle, the tangential velocity component of the particle is the same as that of the gas stream, and the radial gas velocity is neglected.

Gas Flow Pattern: The tangential gas velocity profile for effective separation region was obtained by Crawford [9], with combining Newton's second law and Bernoulli's equation as follows:

$\mathrm{v}_{\theta}=\frac{\mathrm{Q}}{\mathrm{rB} \ln \left(\mathrm{r}_{2} / \mathrm{r}_{1}\right)}$

According to the geometrical relationship in Fig.1, we have $r_{1}=R-B / c$ and $r_{2}=R+B / 2$.

Particle Trajectory: Under the initial assumptions, the 


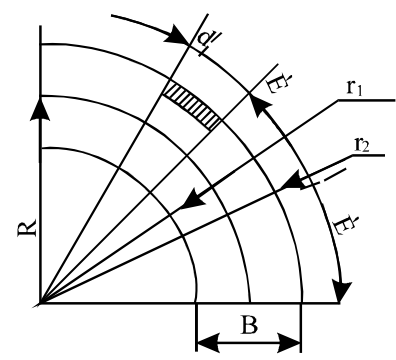

Fig. 1: Cross Section of the Cyclone with Schematic Separation Process

force balance acting on the particle (the drag force obeys Stokes' law) in the radial direction gives:

$$
\frac{\mathrm{d}^{2} \mathrm{r}}{\mathrm{dt}^{2}}+\frac{18 \mu_{\mathrm{g}}}{\rho_{\mathrm{p}} \mathrm{d}_{\mathrm{p}}^{2} \mathrm{C}_{\mathrm{c}}} \frac{\mathrm{dr}}{\mathrm{dt}}-\frac{\mathrm{v}_{\theta \mathrm{p}}^{2}}{\mathrm{r}}=0
$$

Eq. (2) is not readily solvable. An approximate solution can be obtained by neglecting the second derivative $\mathrm{d}^{2} \mathrm{r} / \mathrm{dt}^{2}$, which is the consequence of the assumption that the particle motion in the radial direction is stationary. Eq. (2) then becomes:

$$
\frac{\mathrm{dr}}{\mathrm{dt}}=\frac{\rho_{\mathrm{p}} \mathrm{d}_{\mathrm{p}}^{2} \mathrm{C}_{\mathrm{c}}}{18 \mu_{\mathrm{g}}} \frac{\mathrm{v}_{\theta \mathrm{p}}^{2}}{\mathrm{r}}
$$

According to the Equation of particle trajectory it is:

$$
\begin{aligned}
& \frac{\mathrm{dr}}{\mathrm{dt}}=\mathrm{v}_{\mathrm{rp}} \\
& \frac{\mathrm{d} \theta}{\mathrm{dt}}=\frac{1}{\mathrm{r}} \mathrm{v}_{\theta \mathrm{p}}
\end{aligned}
$$

Combining Eqs. (4) and (5):

$$
\frac{\mathrm{dr}}{\mathrm{d} \theta}=\mathrm{r} \frac{\mathrm{v}_{\mathrm{rp}}}{\mathrm{v}_{\theta \mathrm{p}}}
$$

From Eq. (3), (4) and (6):

$$
\frac{\mathrm{dr}}{\mathrm{d} \theta}=\left(\frac{\rho_{\mathrm{p}} \mathrm{d}_{\mathrm{p}}^{2} \mathrm{C}_{\mathrm{c}}}{18 \mu_{\mathrm{g}}}\right) \mathrm{v}_{\theta \mathrm{p}}
$$

According to the assumption of equal gas and particle velocity, Eqs. (1) and (7) can be combined:

$$
\frac{\mathrm{dr}}{\mathrm{d} \theta}=\left(\frac{\rho_{\mathrm{p}} \mathrm{d}_{\mathrm{p}}^{2} \mathrm{C}_{\mathrm{c}}}{18 \mu_{\mathrm{g}}}\right)\left(\frac{\mathrm{Q}}{\mathrm{B} \ln \left(\mathrm{r}_{2} / \mathrm{r}_{1}\right)}\right) \frac{1}{\mathrm{r}}
$$

Integrating Eq. (8) and considering the boundary condition $\theta_{0}=0, r_{0}=r_{1}$ yields the formula for calculating the trajectory of a particle in any position:

$$
\theta=\left(\frac{18 \mu_{\mathrm{g}}}{\rho_{\mathrm{p}} \mathrm{d}_{\mathrm{p}}^{2} \mathrm{C}_{\mathrm{c}}}\right)\left(\frac{\mathrm{B} \ln \left(\mathrm{r}_{2} / \mathrm{r}_{1}\right)}{\mathrm{Q}}\right)\left[\frac{1}{2}\left(\mathrm{r}^{2}-\mathrm{r}_{1}^{2}\right)\right]
$$

Concentration Distribution: To describe the particle concentration distribution, it is necessary to define the specified point of critical particle size. The particle size distribution is supposed to obey the log-normal distribution:

$f\left(\ln d_{p}\right)=\frac{1}{\ln \sigma \sqrt{2 \pi}} \exp \left[-\frac{\left(\ln d_{p}-\ln d_{p 50}\right)^{2}}{2(\ln \sigma)^{2}}\right]$

According to the equation of particle track, the critical particle size in any position should also be expressed as a function of $r$ and $\theta$ :

$d_{p c}=\sqrt{\left(\frac{18 \mu_{\mathrm{g}}}{\rho_{\mathrm{p}} \mathrm{C}_{\mathrm{c}}}\right)\left(\frac{\mathrm{B} \ln \left(\mathrm{r}_{2} / \mathrm{r}_{1}\right)}{\mathrm{Q}}\right)\left(\frac{1}{\theta}\right)\left[\frac{1}{2}\left(\mathrm{r}^{2}-\mathrm{r}_{1}^{2}\right)\right]}$

Assuming that particles larger than $\mathrm{d}_{\mathrm{pc}}$ can be separated at the specified point and particles less than $d_{p c}$ can not be separated, the particle concentration at this point should be obtained:

$\mathrm{C}(\mathrm{r}, \theta)=\mathrm{C}_{0}\left[1-\int_{\ln \left(\mathrm{d}_{\mathrm{pc}}\right)}^{+\infty} \mathrm{f}\left(\ln \mathrm{d}_{\mathrm{p}}\right) \mathrm{d}\left(\ln \mathrm{d}_{\mathrm{p}}\right)\right]$

We call this the critical separation theory.

Separation Efficiency: The gas-particle separation process in a horizontal cross section of the bends is also schematically shown in Fig. 1. The number of particles per unit volume $\mathrm{C}$ is a function of $\mathrm{r}$ and $\theta$. Suppose that during the time $d_{t}$ all particles, whose distance to the cyclone wall is $d_{r}$ or less, will be collected, and that meanwhile the particles will travel a distance $\operatorname{rd} \theta$ in the tangential and $\mathrm{dz}$ in the vertical direction. Then the number of collected particles within the control volume will be:

$$
-\mathrm{dN}=\mathrm{C}\left(\mathrm{r}_{2}, \theta\right) \mathrm{r}_{2} \mathrm{~d} \theta \mathrm{dzdr}
$$

The total number of particles within the control volume will be:

$$
\mathrm{N}=\int_{\mathrm{r}_{\mathrm{i}}}^{\mathrm{r}_{2}}[\mathrm{C}(\mathrm{r}, \theta) \mathrm{rd} \theta \mathrm{dz}] \mathrm{dr}
$$

According to the boundary layer separation theory, the fraction of particles removed within the control volume can be obtained by combining Eqs. (13) and (14):

$$
-\frac{\mathrm{dN}}{\mathrm{N}}=\frac{\mathrm{C}\left(\mathrm{r}_{2}, \theta\right) \mathrm{r}_{2} \mathrm{dr}}{\int_{\mathrm{r}_{1}}^{\mathrm{r}_{2}} \mathrm{C}(\mathrm{r}, \theta) \mathrm{rdr}}
$$

Near the wall, the Equation of track is:

$$
\frac{\mathrm{dr}}{\mathrm{d} \theta}=\left(\frac{\rho_{\mathrm{p}} \mathrm{d}_{\mathrm{p}}^{2} \mathrm{C}_{\mathrm{c}}}{18 \mu_{\mathrm{g}}}\right) \mathrm{v}_{\theta 2}
$$


Combining and integrating the above Eq. leads to:

$$
-\frac{d N}{N}=\left(\frac{\rho_{p} d_{p}^{2} C_{c}}{18 \mu_{g}}\right) \frac{C\left(r_{2}, \theta\right) r_{2} v_{\theta 2} d \theta}{\int_{r_{1}}^{r_{2}} C(r, \theta) r d r}
$$

Then the particle separation efficiency is:

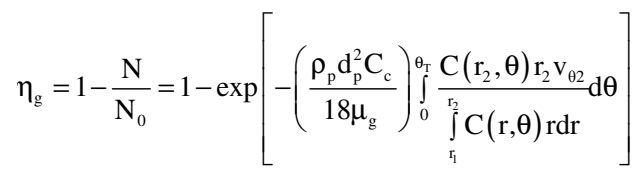

Here, $\theta_{\mathrm{T}}$ is bend angle.

\section{RESULTS AND DISCUSSION}

Effect of Curvature Ratio: Results are shown on the effect of curvature ratio on particle separation efficiency for a range of particle size (Fig. 2). The results indicate that increasing the curvature ratio improves the particle separation efficiency although this difference is insignificant. For example, at a particle size of $6.82 \mu \mathrm{m}$, a bend with a curvature ratio of 1.0 has separation of $43.22 \%, 2.0$ has $47.23 \%$, and 4.0 provides $48.00 \%$. This suggests that a bend with a smaller curvature ratio should be satisfactory for particle collection.

Effect of Bend Angle: The effect of bend angle on particle separation efficiency is illustrated in Fig. 3. The results indicate that increasing the bend angle also improves the particle separation efficiency. However, the efficiency of particle through a $180^{\circ}$ bend should nor be the product of the efficiency of two $90^{\circ}$ bends or four $45^{\circ}$ bends in series. This suggests the there is a non-linear relationship in the various bend angle.

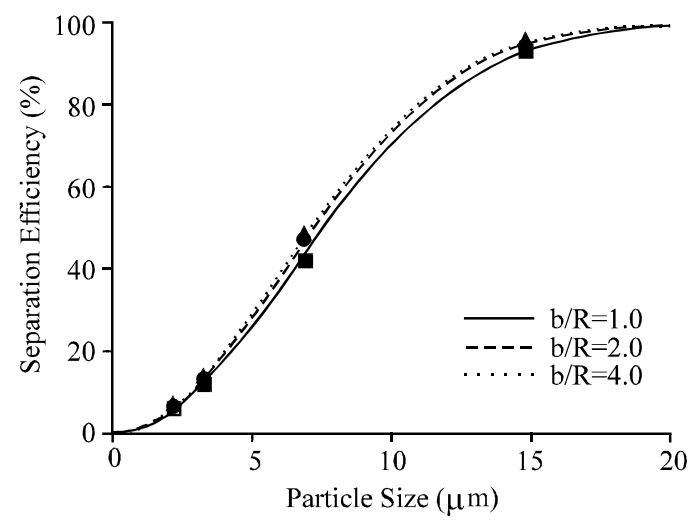

Fig. 2: The Effect of Curvature Ratio on Particle Separation

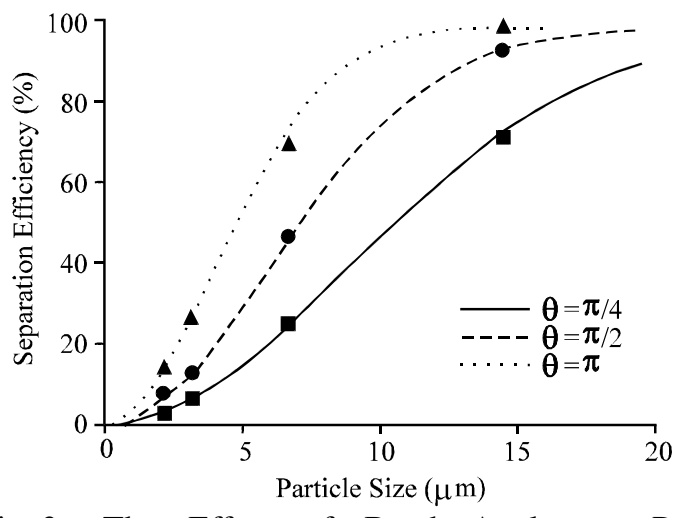

Fig. 3: The Effect of Bend Angle on Particle Separation

\section{CONCLUSION}

A theoretical method was proposed to estimate the particle separation efficiency in bends of rectangular cross-section. Although the new model does not take into account the effects of inlet particle load, the work can serve as a staring point for the development of a new approach to calculate the particle separation efficiency. Further work on this subject is required in order to enhance the adaptability of the model in practice.

\section{REFERENCES}

1. Langdahl, H.D. and R.G.J. Herrmann, 1949. A model for deposition of aerosol particles in a bend. Colloid Sci., 4: 103-107.

2. Davis, R.E., 1964. Secondary flow in bend pipe. Int. J. Air Water Pollut., 8: 177-182.

3. Cheng, Y.S. and C.S. Wang, 1975. Inertial deposition of particles in a bend. J. Aerosol Sci., 6: 139-145.

4. Crane, R.I. and R.L. Evans, 1977. Inertial deposition of particles in a bend pipe. J. Aerosol Sci., 8: 161-170.

5. Cheng, Y.S. and C.S. Wang, 1981. Motion of particles in bends of circular pipes. Atmos. Environ., 15: 301-306

6. Pui, D.Y.H., H.F. Romay-Novas and B.Y.H. Liu, 1987. Experimental study of particle deposition in bends of circular cross-section. Aerosol Sci. Technol., 7: 301-315.

7. Tsai, C.J., D.Y.H. Pui and B.Y.H. Liu, 1990. Capture and rebound of small particle upon impact with solid surfaces. Aerosol Sci. Technol., 12: 197-507.

8. McFarland, A.R., H. Gong, A. Muyshondt, W.B. Wente and N.K. Anand, 1997. Aerosol deposition in bends with turbulent flow. Environ. Sci. Technol., 31: 3371-3377

9. Crawford, M., 1976. Air Pollution Control Theory. McGraw-Hill, New York, USA. 